Research Paper

\title{
The anti-tumor effects of dual PI3K/mTOR inhibitor BEZ235 and histone deacetylase inhibitor Trichostatin A on inducing autophagy in esophageal squamous cell carcinoma
}

\author{
Ning $\mathrm{Wu}^{1{ }^{\circledR *}}$, Yingfeng $\mathrm{Zhu}^{2^{*}}$, Xiao $\mathrm{Xu}^{3^{*}}$, Yongjun $\mathrm{Zhu}^{1}$, Yang Song ${ }^{1}$, Liewen Pang ${ }^{1}$, Zhiming Chen ${ }^{1 凶}$ \\ 1. Department of Cardio-thoracic Surgery, HuaShan Hospital of Fudan University, Shanghai, 200040, China. \\ 2. Department of Pathology, HuaShan Hospital of Fudan University, Shanghai, 200040, China. \\ 3. Central laboratory, HuaShan Hospital of Fudan University, Shanghai, 200040, China. \\ *These authors contributed equally to this work. \\ $\square$ Corresponding author: Tel: +86-21-13564215436 Email: wuning@fudan.edu.cn (N Wu); Tel: +86-21-52887075, Email: czm_md@163.com (Z-M Chen) \\ (c) Ivyspring International Publisher. This is an open access article distributed under the terms of the Creative Commons Attribution (CC BY-NC) license \\ (https://creativecommons.org/licenses/by-nc/4.0/). See http://ivyspring.com/terms for full terms and conditions.
}

Received: 2017.09.18; Accepted: 2018.01.24; Published: 2018.02.28

\begin{abstract}
The effect and regulation of autophagy-related proteins Beclin- 1 and LC3 in esophageal squamous cell carcinoma have not been fully studied. The aim of this study was to assess the expression of Beclin-1 and LC3 in ESCCs, and to investigate the association between the two markers and clinicopathological characteristics as well as prognosis. Meanwhile, we explored the anti-tumor effect of the PI3K/mTOR dual inhibitor BEZ235 and the histone deacetylase inhibitor TSA on inducing autophagy in ESCC cells. Our study included 118 ESCC tumors and paired non-tumor esophageal mucosa tissues. Beclin-1 and LC3 expression were performed by immunohistochemistry. Human ESCC cells Eca-109 and TE-1 were treated with BEZ235 and TSA either alone or in combination in Vitro. The expression of both Beclin-1 and LC3 proteins were decreased significantly in ESCCs, but there was no significant relation between the expression of Beclin- 1 and LC3 $(P=0.427)$. The negative expression of either Beclin-1 or LC3 was associated with advanced TNM stages $(P=0.006$ and $P<0.001$, respectively). Patients with a high expression of Beclin- 1 and LC3 predict better prognosis. In Vitro co-treatment with BEZ235 and TSA showed a synergistic effect on inhibition of ESCC cell viability and induction of autophagy with the increasing expressions of Beclin-1, LC3-II and the ratio of LC3-II/LC3-I. Our results demonstrated that the autophagy-related proteins Beclin-1 and LC3 were decreased in ESCCs and the low expression of the two markers predicted a worse prognosis. The co-treatment of BEZ235 and TSA significantly induced autophagy and enhanced anti-tumor activities, provided a new effective therapeutic target in ESCCs.
\end{abstract}

Key words: Esophageal squamous cell carcinoma; PI3K/mTOR inhibitor; Histone deacetylase inhibitor; angiogenesis; Prognosis.

\section{Introduction}

Esophageal cancer is the eighth most common cancer and the sixth leading cause of cancer-related mortality worldwide. Globally, the number of new cases of esophageal cancer is about 480,000 and more than 400,000 deaths are attributable to this malignancy ${ }^{1}$. Esophageal squamous cell carcinoma (ESCC) is the dominant histological subtype in China $^{2}$. Patients with esophageal cancer had a very poor prognosis because of the high rate of postoperative complications, recurrence and distant 
metastasis $^{3}$. Although therapeutic approaches in targeted esophageal cancer have been improved in recent years, the 5 -year survival rate is still about $40 \%$ by our previous studies 4,5 . The process of esophageal carcinogenesis is a multi-step process. Both the disturbances of signal transduction pathways and the dysfunction of programmed cell death machinery have been demonstrated to be critical for cancer cells to survive and metastasis.

Programmed cell death (PCD) is a regulated cell death mediated by an intracellular program. Apoptosis, also called type I PCD, is characterized by nuclear fragmentation and chromosomal DNA fragmentation. Autophagy, type II PCD, is a vacuolar process of cytoplasmic degradation and characterized by autophagic vacuoles accumulating without apoptosis body forming and no chromosome condensing or caspase independence ${ }^{6}$. The molecular mechanism of autophagy involves several conserved Atg (autophagy-related) proteins. Beclin-1 (mammalian counterpart of the yeast Atg6) and LC3-II (mammalian homologues of yeast Atg8) are thought to be markers of autophagy. The decreased expression of Beclin-1 and LC3 had been found in gastric, lung and breast cancer and predicted the poor prognosis ${ }^{7-9}$. But there is still some controversy regarding the expression of Beclin-1 and LC3 proteins and their inhibitory effect on esophageal cancer. Some studies indicated that the decreased expression of Beclin-1 protein in ESCCs compared to normal esophageal epithelial cells and is correlated with a poor prognosis ${ }^{10}$. Conversely, a few studies had reached the opposite conclusions ${ }^{11}$. Therefore, the expression of Beclin-1 and LC3 in ESCCs has not been fully studied. The aim of our study is to investigate the expression of Beclin-1 and LC3 in human ESCC tissues, and their clinical pathological characteristics as well as prognosis.

Molecular profiling and genomics studies have revealed that the phosphoinositide 3-kinase/ mammalian target of rapamycin (PI3K/mTOR) signaling pathway has been demonstrated to be activated in esophageal cancer ${ }^{12,13}$. The PI3K/mTOR pathway is a critical regulator of autophagy induction $^{14}$, which activated mTOR suppresses autophagy and negative regulation of mTOR promotes it.

Histone deacetylases (HDACs) plays a critical role in the regulation of gene expression and has been shown to contribute to carcinogenesis by inducing the aberrant transcription of key genes regulating important cellular functions such as cell proliferation, cell-cycle regulation and $\mathrm{PCD}^{15}$. Inhibitors of HDACs exert anticancer activity by promoting acetylation of histones as well as by promoting acetylation of non-histone protein substrates, and represent promising potential targets for esophageal cancer treatment ${ }^{16}$. There are two main groups of mammalian HDACs, based on their homology with yeast counterparts. Class I, is homologous to Rpd3 in yeast, includes HDACs 1, 2, 3, and 8, has a nuclear localization, is ubiquitously expressed in human cell lines and tissues. Class II, contains HDAC 4, 5, 6, 7, 9, 10 , is homologous to yeast Hda1, has the nucleus and cytoplasm expression. Both Class I and II HDACs are sensitive to the classical HDAC inhibitor trichostatin A (TSA).

Previous studies suggested that combined intervention of the PI3K/mTOR inhibitors and HDAC inhibitors could be more effective than single drugs in several human cancer cells, such as breast cancer, head and neck squamous cell carcinoma and NSCLC 17-19. However, similar investigation of combining $\mathrm{PI} 3 \mathrm{~K} / \mathrm{mTOR}$ inhibitor with HDAC inhibitor in esophageal cancer has not yet been reported. Thus, our study will investigate the anti-tumor effect of combination of PI3K/mTOR dual inhibitor BEZ235 and HDAC inhibitor TSA on the induction of apoptosis and autophagy in human ESCC cells.

\section{Materials and methods}

\section{Patients and tissue samples}

We selected an esophageal squamous cell carcinoma tissue and a paired non-tumor esophageal mucosa sample from 118 patients who had ESCC and underwent esophagectomy and lymph node dissections at the department of cardio-thoracic surgery of the Huashan Hospital during Jan 1, 2011 to Dec 31, 2013. Patients, who had palliative resections, underwent neoadjuvant or R1/R2 resections were excluded from the analysis. clinicopathologic characteristics as well as prognosis factors were collected. The institutional review board of Huashan hospital, Fudan University reviewed and approved the study.

\section{Clinical data and follow up}

Routine preoperative staging included fibro-gastroscopy, computed tomography of the chest and abdomen, ultrasound imaging of the abdomen and neck and PET-CT scanning. Preoperative nutritional risk assessment, cardiac function tests and pulmonary function test were also performed in all patients to make sure they had the physiological ability to undergo esophagectomy.

Esophageal cancer patients were staged according to the 8th edition AJCC staging system ${ }^{20}$. The standard follow up schedule contained: patients were followed every three months for 2 years, every 
six months for the next three years, and then annually. The outcome is all-cause mortality and defined as time from the data of surgery to the data of death. Routine postoperative tests contained fibrogastroscopy, ultrasound, CT/PET-CT and radionuclide bone scan.

\section{Cell culture and Reagents /antibodies}

Human esophageal carcinoma cancer cell lines Eca-109 and TE-1 were obtained from the Cell Bank of Type Culture Collection of the Chinese Academy of Sciences, Shanghai Institute of Cell Biology (Shanghai, China). Eca-109 and TE-1 cells were cultured in PRMI 1640 medium (GIBCO BRL, Grand Island, NY, USA) and supplemented with $10 \%$ fetal bovine serum (FBS; GIBCO BRL), $100 \mathrm{U} / \mathrm{mL}$ penicillin and $100 \mathrm{U} / \mathrm{mL}$ streptomycin, and incubated at $37^{\circ} \mathrm{C}$ in an atmosphere of $95 \%$ air and $5 \% \mathrm{CO} 2$.

BEZ235 and TSA were purchased from Selleckchem (Houston, TX, USA). The antibodies against Beclin-1, LC3, p-Akt (Ser473), Akt, p-mTOR (Ser2448), mTOR, Bcl-2, p70S6K, p-p70S6K, Caspase-3, cleaved Caspase-3 and $\beta$-actin were purchased from Santa Cruz Biotechnology (Santa Cruz, CA, USA).

\section{Immunohistochemistry (IHC)}

The sample-containing paraffin was sliced into serial sections with a width of $5.0 \mu \mathrm{m}$ for immunohistochemical staining. Each section was deparaffinized for 1 hour at $60^{\circ} \mathrm{C}$ in xylene and rehydrated in serial graded ethanol before being stored overnight in citrate buffer $(0.01 \mathrm{M}, \mathrm{pH} 6.0)$ at $75^{\circ} \mathrm{C}$ for antigen retrieval. Endogenous peroxidase activity was blocked with $0.3 \%$ hydrogen peroxide in methanol. The sections were incubated at $4^{\circ} \mathrm{C}$ overnight with a primary antibody (anti-LC3, mouse monoclonal antibody, 1:400 dilution and anti-Beclin-1, rabbit polyclonal antibody, 1:80 dilutions) and then recovered at $37^{\circ} \mathrm{C}$ for 20 minutes. The slides were incubated with the secondary antibody for 30 minutes at room temperature and then were developed by DAB. Nuclear staining was carried out with hematoxylin. Normal esophageal tissue was serving as the negative controls.

\section{Evaluation of IHC staining}

LC3 and Beclin-1 were considered positive by cytoplasmic and cytomembrane staining. The results were interpreted by two independent pathologists who were blinded to the specific diagnosis and prognosis for each case. For each sample, at least five fields were chosen randomly and at least 500 cells were required for scoring in each field. Staining extent of immunostaining was scored according to the percentage of positive stained cells as follows: 0 , none;
1, up to $10 \%$ positive cells; $2,10 \%-50 \%$ positive cells; $3,51 \%-80 \%$ positive cells; 4 , positive cells $>80 \%$. The intensity of staining was as follows: 0 , none; 1 , low-intensity (light yellow); 2, moderate-intensity (yellow); and 3, strong-intensity (reddish brown). The score was calculated as grade in stain intensity* ${ }^{*}$ grade in coloration rate, which ranging from 0 to 12 . The scores $\geq 4$ were defined as positive over-expression. Low expression group included negative expression or low expression samples with overall score $0-3$. Both the esophageal cancer tissue and the normal esophageal tissue use the same scoring criteria.

\section{Cell viability assay}

Cell viability was measured using the MTT [3-(4, 5-Dimethylthiazol-2-yl)-2, 5- diphenyl tetrazolium] assay and colony formation test. Eca-109 cells were cultured in 96-well plates to adhere overnight, and were incubated $24 \mathrm{~h}$ prior to treatment. The cells were treated with $\operatorname{BEZ} 235(0,50,100,200,300,500$ and 800 $n M)$, TSA $(450 \mathrm{nM})$ or BEZ235 $(150 \mathrm{nM})+$ TSA (450 $\mathrm{nM})$ for 24-48 hours. Untreated cells were defined as controls. MTT $(5 \mathrm{mg} / \mathrm{mL})$ was added to each well followed by 4 -hour incubation. $100 \mu \mathrm{L}$ DMSO was added to dissolve the resulting formazan crystals. Absorbance values at $490 \mathrm{~nm}$ were determined. The experiments were repeated at least 3 times.

\section{Western blot analysis}

Western blot analysis was carried out as we described previously, Target proteins were fractionated by SDS-PAGE and then electro-transferred into polyvinylidene difluoride membranes (Sigma, St. Louis, MO, USA). The membrane was blocked with 5\% nonfat dry milk for 1 $\mathrm{h}$ at room temperature and subsequently incubated primary antibodies against p-Akt, Akt, p-mTOR, mTOR, Bcl-2, p70S6K, p-p70S6K, Caspase-3, cleaved Caspase-3, Beclin- 1 and LC3 overnight at $4^{\circ} \mathrm{C}$. After washing, the membranes were incubated with an anti-rabbit horseradish peroxidase antibody for $1 \mathrm{~h}$ at room temperature. The specific bands on the blots were visualized using a chemiluminescence detection kit (Thermo Fisher Scientific, Waltham, MA, USA). Protein expression levels were normalized to $\beta$-actin.

\section{Statistical analysis}

The associations between IHC expression and clinicopathological variables were examined using $x^{2}$ test and Fisher's exact test for categorical variables and the Student's $t$ test for continuous variables. The Kaplan-Meier survival curve and log-rank tests were performed to estimate the survival function across groups. Cox's proportional hazard regression model for multivariate analysis was performed to identify 
the independent prognostic factors. Vitro studies for comparison of multiple groups are presented as mean $\pm \mathrm{SD}$ for at least three times. Unpaired t-tests were used for analyzing different variables (BEZ235 and TSA). Paired t-test analysis was used to compare different time points of the same variable (before and after treated with drug). Statistical analyses were performed using SPSS 16.0 software (SPSS, Inc., Chicago, USA). Significance was defined as $P<0.05$.

\section{Results}

\section{Patient Characteristics}

The clinical characteristics and 5-years survival rate are summarized in Table $\mathbf{1}$. There were 98 men and 20 women. The median age was 61 years (range, $38-82$ years). The AJCC stage distribution was as follows: stage I, $\mathrm{n}=22$ (18.6\%); stage II, $\mathrm{n}=48(40.7 \%)$; stage III, $\mathrm{n}=37(31.4 \%)$ and stage IV, $\mathrm{n}=11(9.3 \%)$. The median follow-up time was 43 months.

Table 1. Patient characteristics and univariate analysis

\begin{tabular}{|c|c|c|c|}
\hline Factors & No. & 5-year survival (\%) & $p$ \\
\hline \multicolumn{4}{|l|}{ Age, year } \\
\hline$\leq 65$ & 83 & $45.3 \%$ & 0.662 \\
\hline$>65$ & 35 & $48.8 \%$ & \\
\hline \multicolumn{4}{|l|}{ Sex } \\
\hline Male & 98 & $49.2 \%$ & 0.295 \\
\hline Female & 20 & $35 \%$ & \\
\hline \multicolumn{4}{|c|}{ Tumor location } \\
\hline Upper & 17 & $29.4 \%$ & 0.034 \\
\hline Middle & 68 & $52.3 \%$ & \\
\hline Lower & 33 & $43.7 \%$ & \\
\hline Surgical type & & & 0.107 \\
\hline 2-FL & 60 & $52.3 \%$ & \\
\hline 3-FL & 58 & $41.1 \%$ & \\
\hline \multicolumn{4}{|c|}{ Differentiation } \\
\hline Well & 8 & 87.5 & 0.117 \\
\hline Moderately & 73 & 40.7 & \\
\hline Poorly & 37 & 50.3 & \\
\hline \multicolumn{4}{|l|}{ T stage } \\
\hline $\mathrm{T} 1+\mathrm{T} 2$ & 60 & 66.1 & 0.000 \\
\hline $\mathrm{T} 3+\mathrm{T} 4$ & 58 & 26.3 & \\
\hline \multicolumn{4}{|l|}{ TNM stage } \\
\hline I & 22 & 85.2 & 0.000 \\
\hline II & 48 & 59.7 & \\
\hline III & 37 & 18.1 & \\
\hline IV & 11 & 13.6 & \\
\hline \multicolumn{4}{|l|}{ Beclin-1 } \\
\hline Positive & 68 & 54.5 & 0.007 \\
\hline Negative & 50 & 35.1 & \\
\hline \multicolumn{4}{|l|}{ LC3 } \\
\hline Positive & 57 & 61.3 & 0.021 \\
\hline Negative & 61 & 33.0 & \\
\hline
\end{tabular}

\section{Decreased expression of Beclin-1 and LC3 proteins in ESCCs}

The expression of Beclin-1 and LC3 were assessed in all 118 ESCC tissues and paired normal esophageal mucosa tissues by immunohistochemistry. Beclin-1 staining was observed mainly in the cytoplasm of the esophageal cancer cells while LC3 was observed in the cytoplasm and cytomembrane of cancer cells (Figure 1).

The immunohistochemistry results showed that $57.6 \%(68 / 118)$ and $48.3 \%(57 / 118)$ ESCC cells expressed Beclin-1 or LC3, respectively, $82.2 \%$ $(97 / 118)$ and $73.7 \%(87 / 118)$ cells in paired normal tissues expressed Beclin-1 or LC3. The expression of both Beclin-1 and LC3 proteins were decreased significantly in ESCCs.

\section{Relationship between Beclin-1, LC3 expression and clinicopathological features}

Table 2. Associations of beclin-1 and LC3 expressions with chinicopathologic characteristics in ESCCs.

\begin{tabular}{|c|c|c|c|c|c|c|c|}
\hline \multirow[t]{2}{*}{ Factors } & \multirow[t]{2}{*}{ No } & \multicolumn{2}{|c|}{ Beclin-1 } & \multirow[t]{2}{*}{$p$} & \multicolumn{2}{|l|}{ LC3 } & \multirow[t]{2}{*}{$p$} \\
\hline & & $\mathrm{n}(+)$ & $\mathrm{n}(-)$ & & $\mathrm{n}(+)$ & $\mathrm{n}(-)$ & \\
\hline \multicolumn{8}{|l|}{ Age } \\
\hline$\leq 65$ & 83 & 50 & 33 & 0.381 & 37 & 46 & 0.216 \\
\hline$>65$ & 35 & 18 & 17 & & 20 & 15 & \\
\hline \multicolumn{8}{|l|}{ Sex } \\
\hline Male & 98 & 54 & 44 & 0.223 & 49 & 49 & 0.419 \\
\hline Female & 20 & 14 & 6 & & 8 & 12 & \\
\hline \multicolumn{8}{|l|}{ Tumor location } \\
\hline Upper & 17 & 11 & 6 & 0.520 & 8 & 9 & 0.434 \\
\hline Middle & 68 & 39 & 29 & & 36 & 32 & \\
\hline Lower & 33 & 18 & 15 & & 13 & 20 & \\
\hline \multicolumn{8}{|l|}{ Surgical type } \\
\hline 2-FL & 60 & 37 & 23 & 0.371 & 31 & 29 & 0.462 \\
\hline 3-FL & 58 & 31 & 27 & & 16 & 32 & \\
\hline \multicolumn{8}{|l|}{ Differentiation } \\
\hline Well & 8 & 4 & 4 & 0.226 & 7 & 1 & 0.750 \\
\hline Moderately & 73 & 47 & 26 & & 28 & 45 & \\
\hline Poorly & 37 & 17 & 20 & & 22 & 15 & \\
\hline \multicolumn{8}{|l|}{ T stage } \\
\hline $\mathrm{T} 1+\mathrm{T} 2$ & 60 & 39 & 21 & 0.101 & 37 & 23 & 0.003 \\
\hline $\mathrm{T} 3+\mathrm{T} 4$ & 58 & 29 & 29 & & 20 & 38 & \\
\hline \multicolumn{8}{|l|}{ TNM stage } \\
\hline I & 22 & 17 & 5 & 0.006 & 17 & 5 & 0.000 \\
\hline II & 48 & 30 & 18 & & 28 & 20 & \\
\hline III & 37 & 15 & 22 & & 8 & 29 & \\
\hline IV & 11 & 6 & 5 & & 4 & 7 & \\
\hline \multicolumn{8}{|l|}{ Comorbidities } \\
\hline Highblood & 60 & 27 & 33 & 0.004 & 28 & 32 & 0.720 \\
\hline Copd & 13 & 9 & 4 & 0.374 & 8 & 5 & 0.316 \\
\hline $\begin{array}{l}\text { Cardiovascular } \\
\text { disease }\end{array}$ & 19 & 11 & 8 & 0.980 & 12 & 7 & 0.160 \\
\hline Arrhythmia & 11 & 6 & 5 & 0.830 & 4 & 7 & 0.410 \\
\hline Diabetes & 40 & 23 & 17 & 0.984 & 17 & 23 & 0.370 \\
\hline \multicolumn{8}{|l|}{ Complications } \\
\hline 90-day mortality & 4 & 1 & 3 & 0.017 & 2 & 2 & 0.946 \\
\hline Fistula & 13 & 4 & 9 & 0.038 & 5 & 8 & 0.456 \\
\hline Sepsis & 11 & 4 & 7 & 0.136 & 6 & 5 & 0.667 \\
\hline Pneumonia & 56 & 32 & 24 & 0.920 & 30 & 26 & 0.281 \\
\hline $\begin{array}{l}\text { cardiac } \\
\text { insufficiency }\end{array}$ & 21 & 12 & 9 & 0.961 & 9 & 12 & 0.585 \\
\hline \multicolumn{8}{|l|}{ mTOR } \\
\hline Positive & 62 & 28 & 34 & 0.005 & 25 & 37 & 0.097 \\
\hline Negative & 56 & 40 & 16 & & 32 & 24 & \\
\hline \multicolumn{8}{|l|}{ LC3 } \\
\hline Positive & 57 & 35 & 22 & 0.427 & & & \\
\hline Negative & 61 & 33 & 28 & & & & \\
\hline
\end{tabular}




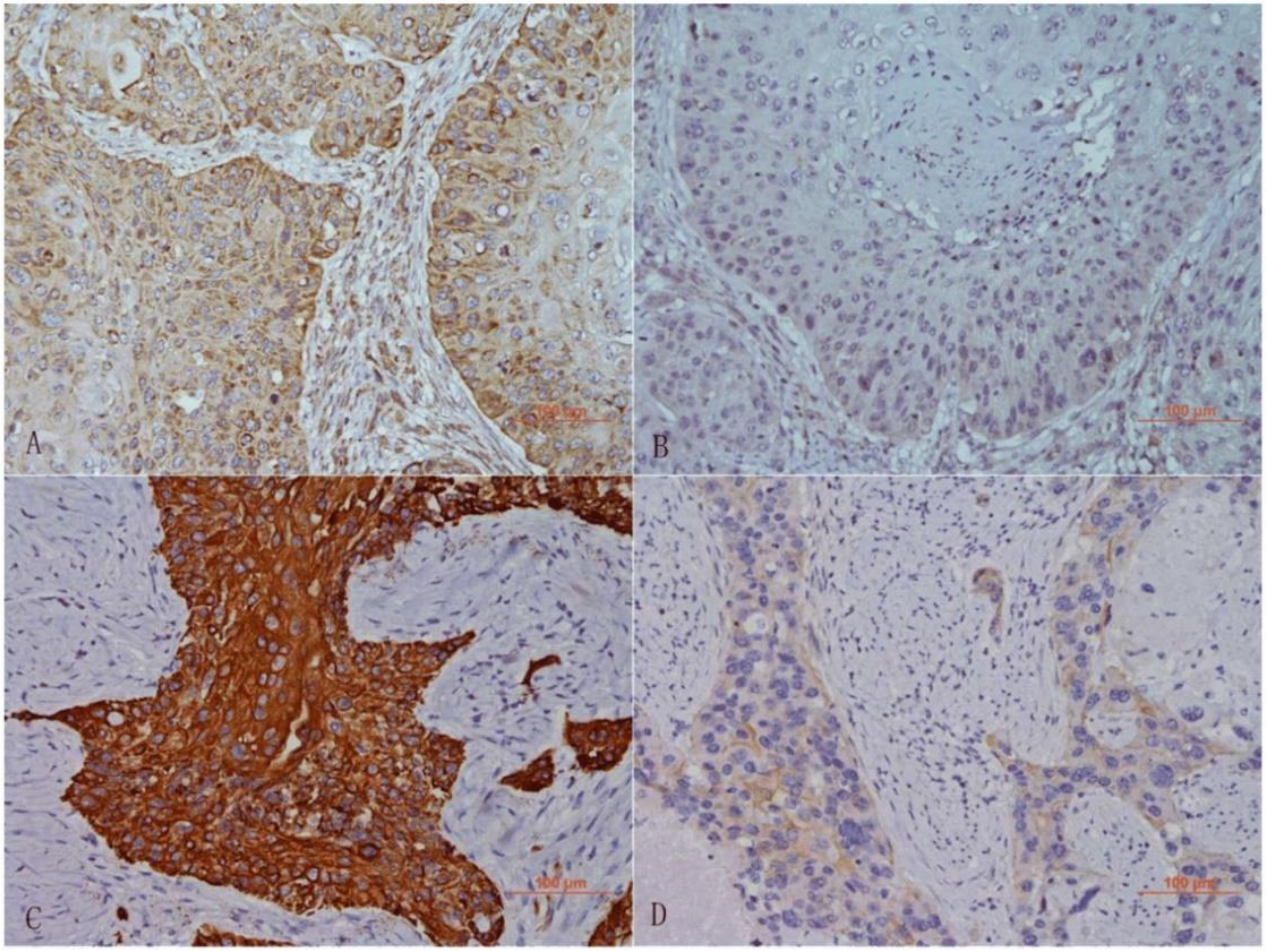

Figure 1. Immunohistochemical analysis of Beclin-1 and LC3 in ESCCs. High expression (A) and low expression (B) of Beclin-1 in cytoplasm of tumor cells. High expression (C) and low expression (D) of LC3 in cytoplasm and cytomembrane of tumor cells. A-D: 20x magnification.

We analyzed the correlations between Beclin-1, LC3 expression and clinical histopathologic parameters (Table 2). Our study showed patients with positively expressed LC3 have a tender to have lower T-stages. Meanwhile, the negative expression of either Beclin-1 or LC3 was associated with advanced TNM stages $(P=0.006$ and $P<0.001$, respectively). Beclin-1 and LC3 protein expression did not show any association with age, gender, tumor location, surgical type and tumor differentiation $(\mathrm{P}>0.05)$.

For major preoperative comorbidities and postoperative complications, Highblood and fistula of esophageal anastomosis appeared to be more frequent in Beclin-1 negative patients.

We analyzed the association between LC3 and Beclin-1 protein expression. In the 68 Beclin-1 positive ESCC patients, 35 also had a positive expression of LC3. However, in the 50 Beclin-1 negative tumors, LC3 was not detected in 28 patients. We did not observe any significant association between LC3 and Beclin-1 expression ( $\mathrm{P}=0.427)$.

Our analysis results showed that Beclin-1 expression was negatively correlated with mTOR expression $(\mathrm{P}=0.005)$. We could also found a tendency towards negative correlat767ion with LC3 and mTOR expression, but there was no significant difference $(\mathrm{p}=0.097)$.

\section{Univariate and multivariate survival analysis}

The overall 1-, 3- and 5-year survival rate was $86.3 \%, 59.3 \%$ and $46.6 \%$, respectively. The potential factors affecting overall survival by univariate analysis were tumor location $(\mathrm{P}=0.034), \mathrm{T}$ stage $(\mathrm{P}<0.001)$, TNM stage $(\mathrm{P}<0.001)$, Beclin-1 expression $(\mathrm{P}=0.007)$ and LC3 expression $(\mathrm{P}=0.021)$, as shown in Table 1. High expression of Beclin-1 and LC3 predict better OS prognosis (Figure 2). Meanwhile, univariate survival analysis for disease-free survival (DFS) also showed similar results, patients with a high expression of Beclin-1 (54.3\% vs. $34.2 \%, p=0.023$ ) or LC3 (58.6 \% vs. $34.1 \%, \mathrm{p}=0.014)$ had a better 5 -year DFS rate.

The multivariate analysis indicated TNM stage and Beclin-1 expression (HR $=0.511 ; \quad 95 \%$ CI: 0.299-0.874, $\mathrm{P}=0.014$ ) were significant risk factors (Table 3). The expression of Beclin-1 was proved to be an independent prognostic factor for overall survival in ESCC patients.

Table 3. Multivariate prognostic analyses of overall survival

\begin{tabular}{llll}
\hline Prognostic factors & $P$ values & HR & $95 \%$ CI \\
\hline Tumor location & 0.291 & 0.781 & $0.494-1.236$ \\
T-stage (T1+2 vs T3+4) & 0.449 & 1.297 & $0.662-2.542$ \\
TNM-stage & 0.001 & 1.893 & $1.293-2.773$ \\
Beclin-1 & 0.014 & 0.511 & $0.299-0.874$ \\
LC3 & 0.526 & 1.201 & $0.681-2.118$ \\
\hline
\end{tabular}



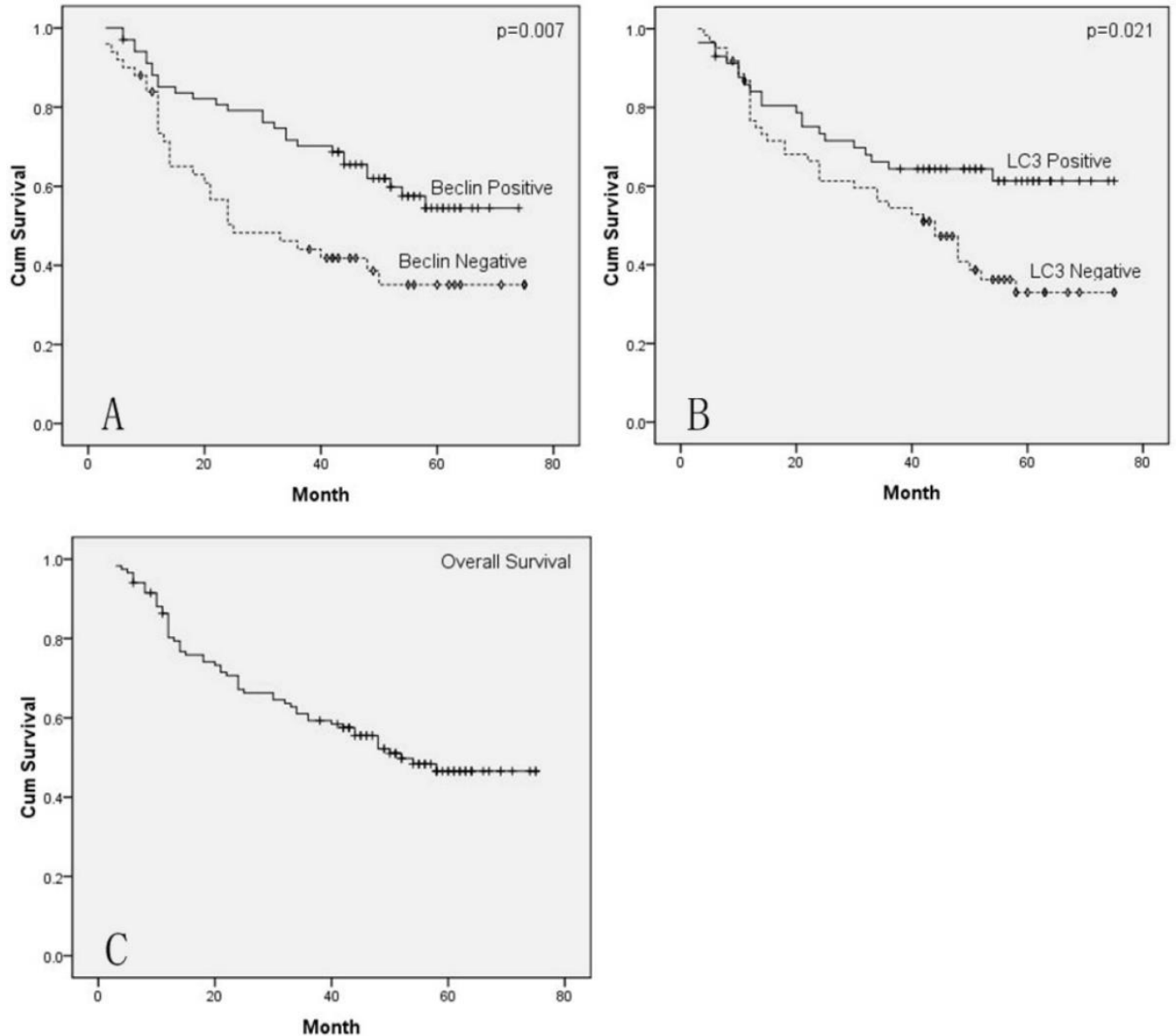

Figure 2. Cumulative Kaplan-Meier survival curves. A. Curves of OS for patients with Beclin-1 positive and Beclin-1 negative tumors. B. Curves of OS for patients with LC3 positive and LC3 negative tumors. C. Curves of OS for all patients.
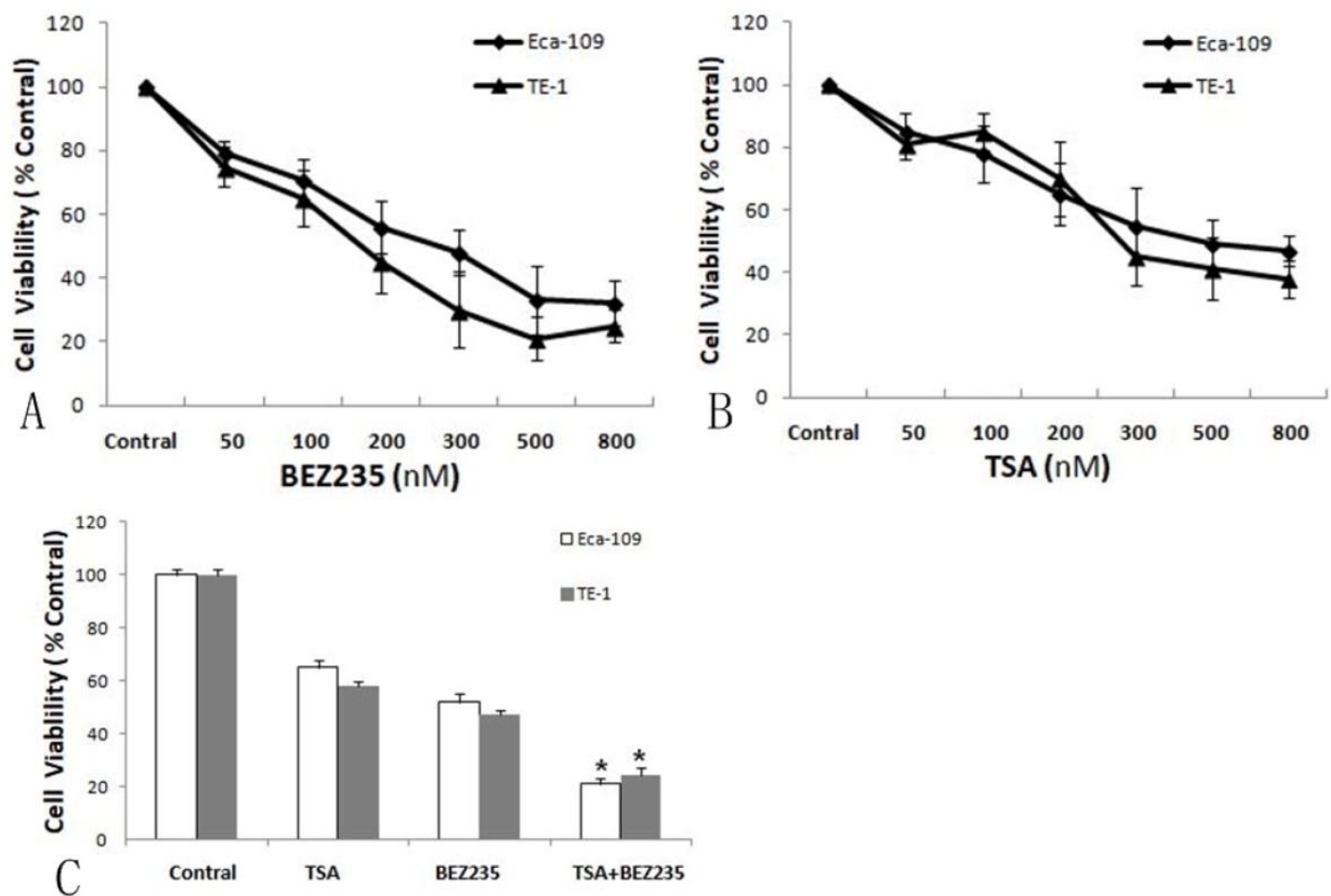

Figure 3. Cell viability of Eca-109 and TE-1 cells, treated by either BEZ235 (A) or TSA (B) for 48h, was measured by MTT assay. C. Co-treatment of BEZ235 and TSA showed synergistically cytotoxic effect on ESCC cells. Each cell was analyzed in three times. ${ }^{*} p<0.05$ compared with control by t-test. 


\section{Co-treatment with BEZ235 and TSA inhibits cell viability on Eca- 109 and TE- 1 cells}

To examine the inhibitory effect of BEZ235 and TSA on the proliferation of Eca-109 and TE-1 cells, we evaluated the cell viability before and after the increasing concentrations of BEZ235 or TSA ranging from 50 to $800 \mathrm{nM}$. As shown in Figure 3, the cell viability was significantly decreased after treatment with BEZ235 or TSA in both Eca-109 and TE-1 cells, indicating that both BEZ235 and TSA had dose dependent anti-tumor effects. The IC50 values for BEZ235 were $160.7 \mathrm{nM}$ and $109.4 \mathrm{nM}$ for Eca-109 and TE-1 cells, respectively. The IC50 values for TSA were $522.3 \mathrm{nM}$ and $374.7 \mathrm{nM}$, respectively. And the two esophageal cancer cells were more sensitive to BEZ235. We set the indicated BEZ235 and TSA concentrations to $150 \mathrm{nM}$ and $450 \mathrm{nM}$ respectively.

\section{Effect of BEZ235 and TSA treatment on PI3K/mTOR pathway}

Firstly, we detected the expression of PI3K/mTOR pathway proteins in Eca-109 cells treated with increasing concentration of BEZ235 (0, 100, 200 and $500 \mathrm{nM}$ ) for 48 hours. The western blot results showed that BEZ235 inhibited the expression of p-mTOR, p-AKT and p-p70S6K in Eca-109 cells $(P<0.05)$. Moreover, the inhibition effect was dose dependent and significantly enhanced with the increasing dose of BEZ235 (Figure 4A).

Then, we detected the PI3K/mTOR pathway proteins after 48 hours of co-treatment of BEZ235 (150 $\mathrm{nM})$ and TSA $(450 \mathrm{nM})$. As shown in Figure 4B, compare to BEZ235, TSA moderately affect the PI3K/mTOR pathway protein phosphorylation. After the combination with BEZ235 and TSA, it significantly inhibited the phosphorylation of mTOR, AKT and p70S6K in both Eca-109 and TE-1 cells relative to single drug.

\section{Co-treatment with BEZ235 and TSA induces apoptosis and autophagy on Eca-109 and TE-1 cells}

To explore whether co-treatment with BEZ235 $(150 \mathrm{nM})$ and TSA (450nM) can induce apoptosis and autophagy, both apoptosis- and autophagy-related proteins were examined by western blot (Figure 5). Eca-109 and TE-1 cells were incubated with BEZ235 and TSA. We detected the apoptotic relative proteins caspase-3, cleaved caspase-3 and BCL-2. Compared to treatment with either drug alone, co-treatment with BEZ235 and TSA for 48 hours, the expression of cleaved caspase-3 was increased as well as the expression of caspase-3 and BCL-2 were decreased. These results suggested that co-treatment with BEZ235 and TSA synergistically induced apoptosis through both the mitochondrial pathway and the caspase-dependent pathway for cell death in ESCC cells.

Compared with treatment with either drug alone, the apoptotic relative proteins LC3-I, LC3-II and Beclin-1 were detected and immunoblotting analysis found co-treatment with BEZ235 and TSA significantly increased the expression of both LC3-II and Beclin-1 in ESCC cells, but the effect on the expression of LC3-I was no obvious (Figure 6). Therefore the co-treatment could also increase the ratio of LC3-II/LC3-I. Our finding indicated that co-treatment with BEZ235 and TSA exhibited anti-cancer effects through not only autophagy but also apoptosis pathways in ESCC cells.
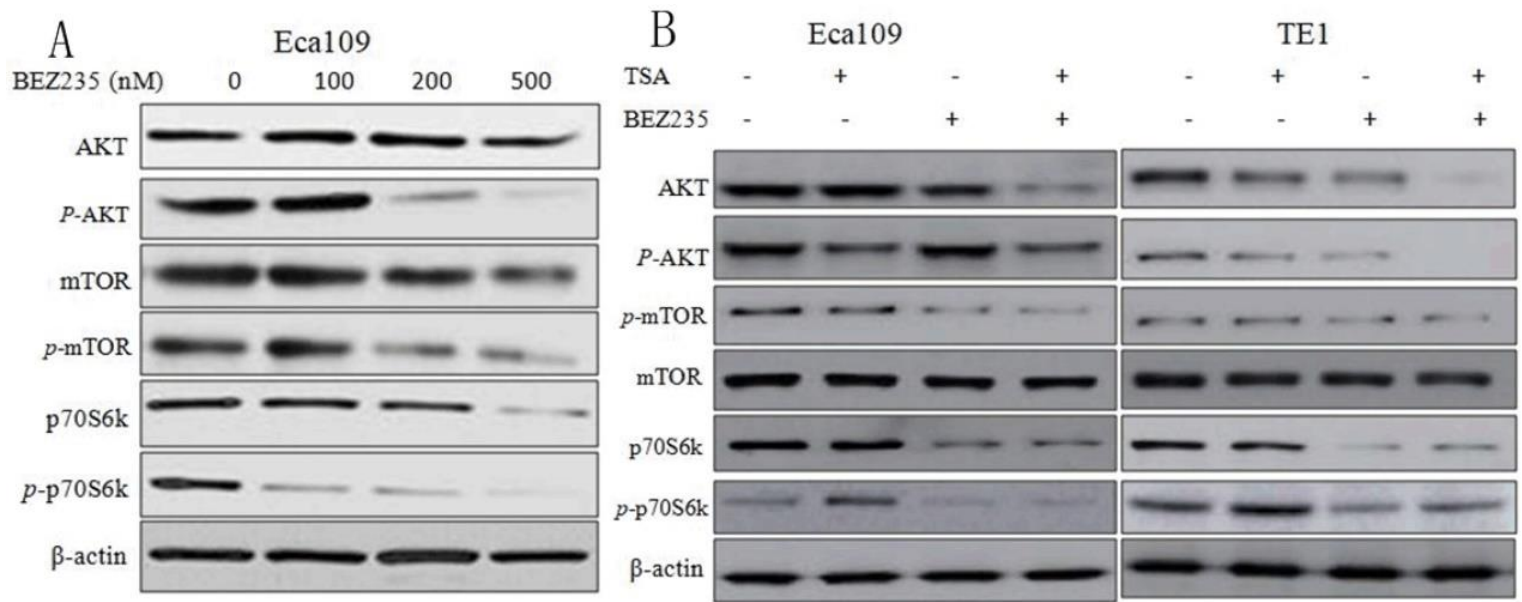

Figure 4. Effect of treatment with BEZ235 or TSA on PI3K/mTOR pathway in ESCC cells. A. Eca-109 cells was treated with increasing concentration of BEZ235 ( 0 , 100, 200 and $500 \mathrm{nM}$ ) for 48 hours. Western blot showed the inhibition effect of PI3K/mTOR pathway was dose dependent. B. Co-treatment of BEZ235 (150 nM) and TSA (450 nM) for 48 hours increased inhibition of the PI3K/mTOR pathway in Eca-109 and TE-1 cells. Each protein was analyzed in three times, and one representative experiment is shown. 


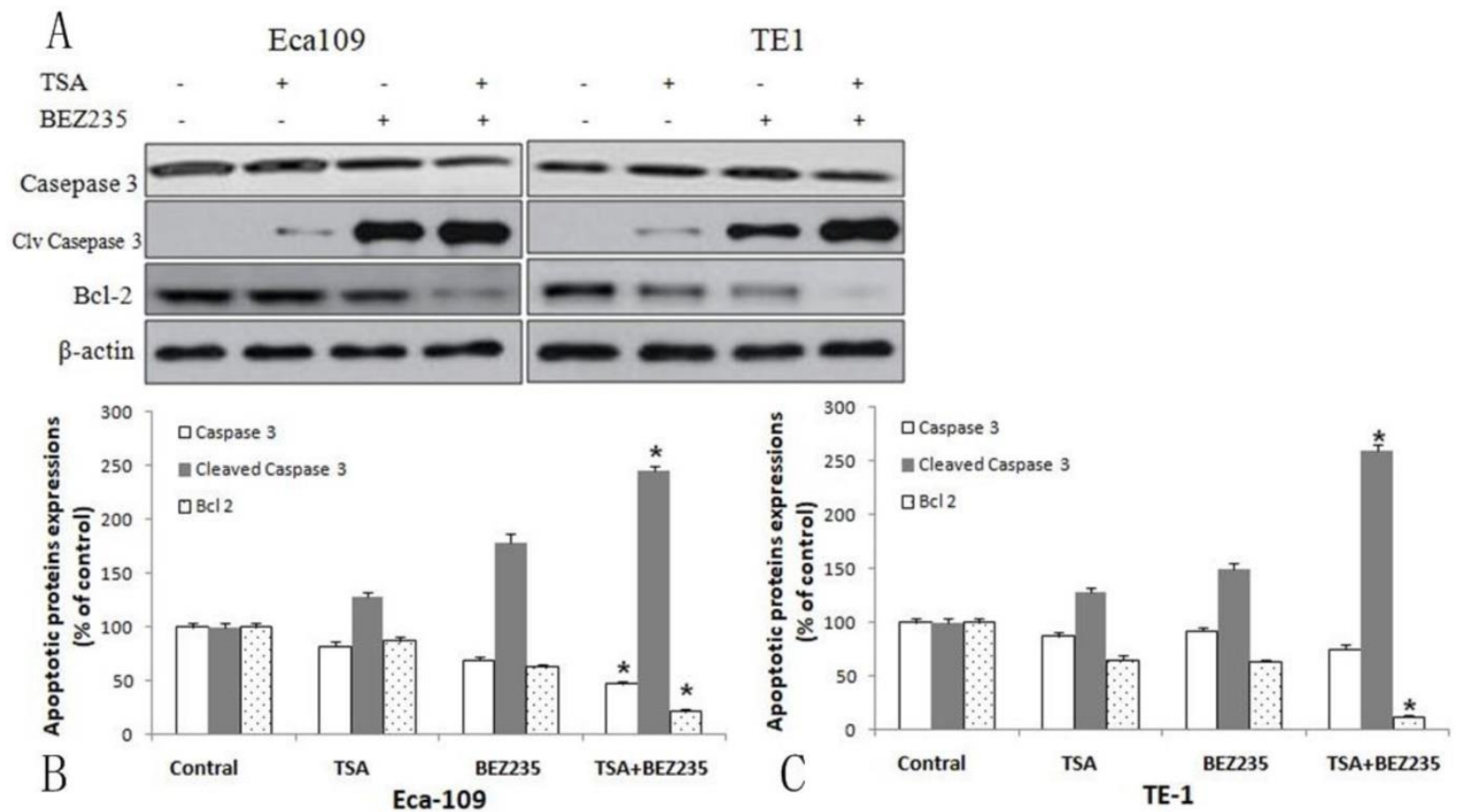

Figure 5. Apoptosis induced by co-treatment with BEZ235 and TSA in ESCC cells. A. Co-treatment of BEZ235 (150 nM) and TSA (450 nM) for 48 hours, western blot showed the expression of cleaved caspase- 3 was increased as well as the expression of caspase-3 and BCL-2 were decreased. Percentage of apoptotic proteins expression of Eca-109 (B) and TE-1 (C) cell, compared to control. *p<0.05 compared with control and single reagent.

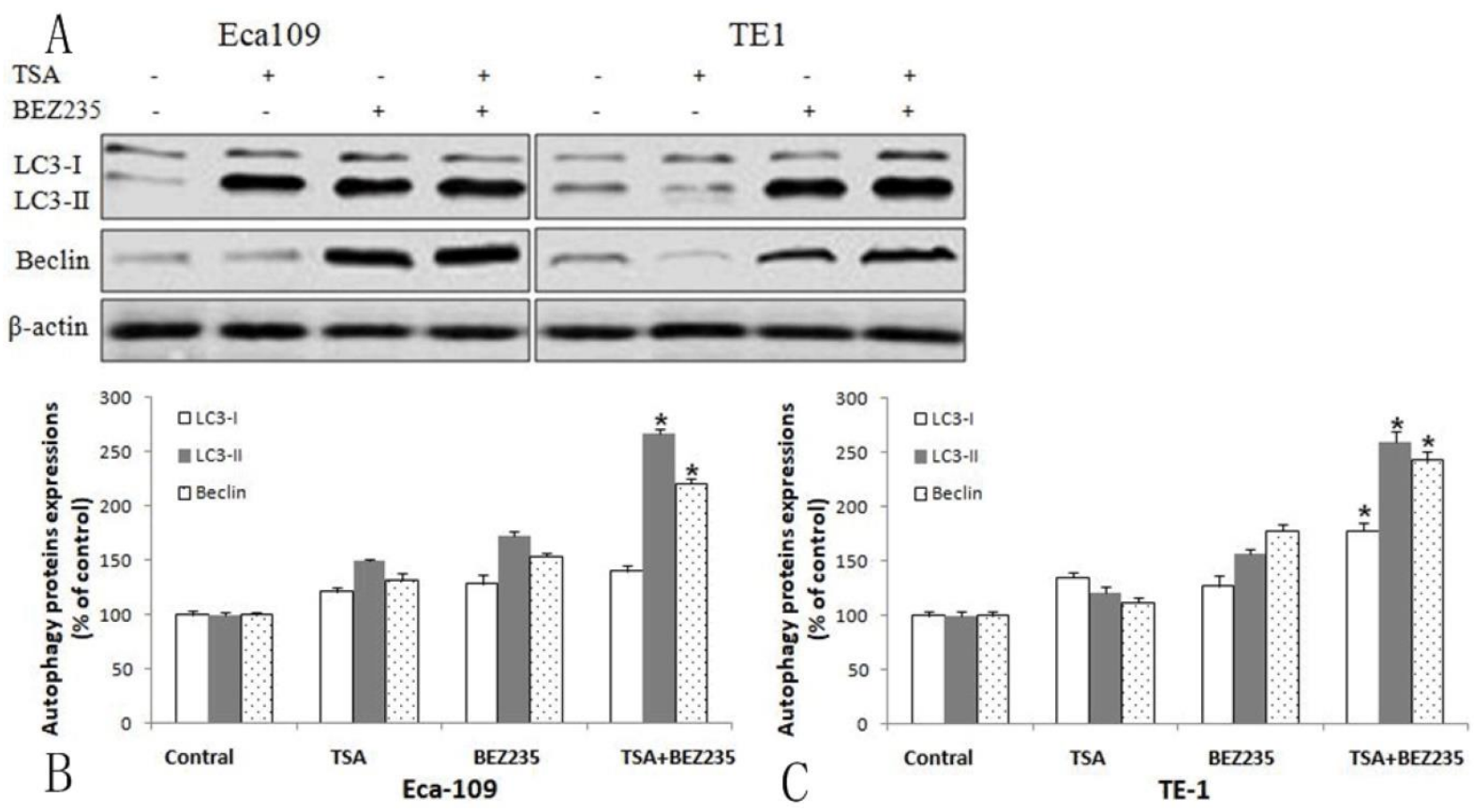

Figure 6. Autophagy induced by co-treatment with BEZ235 and TSA in ESCC cells. A Co-treatment of BEZ235 (150 nM) and TSA (450 nM) for 48 hours, significantly increased the expression of both LC3-II and Beclin-1. Percentage of autophagic proteins expression of Eca-109 (B) and TE-1 (C) cell, compared to control. *p $<0.05$ compared with control and single reagent.

\section{Discussion}

Autophagy is regarded as a non-apoptotic form of PCD or called autophagic cell death and plays a fundamental role in the regulation of cell growth, proliferation, cell survival and cell death. Abnormal regulation of PCD is associated with a wide variety of human diseases, including cancer. And previous evidences showed autophagy is involved in the process of tumorigenesis in various solid tumors ${ }^{21}$.

Beclin-1, the mammalian ortholog of yeast Atg6, is the first identified mammalian autophagy gene and created a core component of the PI3K-III complex. Beclin-1 plays an important role in recruiting proteins 
from the cytoplasm for autophagic degradation or in supplying the autophagic pathway with membrane components and involves in the initial step of autophagosome formation and is shown to be a positive regulator of autophagy and a tumor suppressor ${ }^{22}$. LC3 is an autophagosomal ortholog of yeast Atg8. During autophagy, the lipidated form of LC3 (LC3-II) is recruited to autophagosomal membranes. This conversion is considered to correlate with autophagic activity ${ }^{23}$. Thus, LC3-II has become a reliable marker for monitoring autophagy and autophagy-related processes ${ }^{24}$.

There is a great deal of controversy about the role and effect of the autophagy-related proteins Beclin-1 and LC3 on solid tumor cell proliferation and prognosis. Several studies had found the down-regulated expression of Beclin-1 and LC3 proteins were correlated with poor survival in various tumors. $\mathrm{Yu}$ et al showed Beclin-1 and MAP1LC3B were lower in gastric cancer tissues than adjacent normal tissues and both the MAP1LC3B and Beclin-1 low expression patients had shorter OS time and RFS time ${ }^{7}$. Yang et al found the high expression of Beclin-1 in colorectal cancer and was correlated with histological grade and clinical stage and patients with a high Beclin-1 expression had a better OS and DFS ${ }^{25}$. On the contrary, $\mathrm{Wu}$ et al found the positive expression rates of Beclin-1 and LC3 in colorectal cancer tissues were higher than those in adjacent tissues 26 .

Focusing on esophageal adenocarcinoma (EAC), previous studies almost got the same results that Beclin-1 expression was low in EAC tissues or cell lines 27, 28, and Weh et al found Beclin-1 expression loss occurred in $49.0 \%$ of EAC patients versus $4.8 \%$ of controls and there was a significant inverse correlation between loss of Beclin-1 with histologic grade and tumor stage.

So far the main controversy has focused on the expression and role of the two proteins on ESCCs. YS Chen et al found a loss of Beclin-1 protein expression in $33 \%$ of ESCCs, and the survival rate with Beclin-1 positive patients was significantly higher ${ }^{10}$. But Chen $Y$ et al indicated the over-expression of Beclin1 and LC3 in esophageal cancer, and patients with Beclin1 and LC3 negative expression had a better OS ${ }^{11}$. In order to clarify the role and effect of autophagyrelated proteins on ESCCs, we evaluated the two crucial autophagy-related proteins in 118 ESCC patients and found both Beclin-1 and LC3 expressions were significantly decreased in ESCC tissues compared with normal esophageal epithelial tissues. Meanwhile, our study found both Beclin-1 and LC3 expressions were significantly correlated with the T-stage and the AJCC TNM stage. Patients with a high expression of Beclin-1 or LC3 had a better OS or DFS. These finding indicated that autophagy plays an important role in the development and prognosis of ESCCs. In order to explore the molecular mechanism of autophagy and verify whether the inhibition of autophagy had potential value in anti-cancer therapy in ESCCs.

The PI3K/mTOR pathway dysregulation has been found in various tumors and plays a crucial role in regulation of cell proliferation, apoptosis and autophagy. Our previous studies had found $\mathrm{PI} 3 \mathrm{~K} / \mathrm{mTOR}$ pathway was aberrantly activated in ESCCs, and the present study found both Beclin-1 and LC3 expression had a tendency towards negative correlation with mTOR expression. Thus, the $\mathrm{PI} 3 \mathrm{~K} / \mathrm{mTOR}$ inhibitors are appealing pharmacologic target in esophageal cancer treatment.

Our study found BEZ235, the dual inhibitor of mTOR and PI3K can reduce viability of ESCC cells in a dose-dependent manner caused by the significant reduction in p-Akt, p-mTOR and p-p70S6K activity on Eca-109 and TE-1 cells. After BEZ235 intervention, the autophagy or apoptosis-related proteins beclin-1, LC3-II, ratio of LC3-II/LC3-I and cleaved caspase-3 were increased as well as caspase-3 and BCL-2 decreased. The study reveals that BEZ235 inhibits the $\mathrm{PI} 3 \mathrm{~K} / \mathrm{mTOR}$ pathway and induces the autophagy and apoptosis in human esophageal cancer cells.

Epigenetic mechanisms, such as modifications of DNA-structure or histone modification play particularly important roles in controlling PCD, such as autophagy, apoptosis and necroptosis in tumor cells. The up-regulated of HDAC has been observed in several types of solid tumors, including non-small cell lung cancer, breast cancer and so on ${ }^{17,19}$. HDAC inhibitors cause changes in the acetylation status of chromatin and other non-histone proteins, resulting in changes in gene expression, induction of apoptosis and inhibition of angiogenesis and metastasis. In recent years, more studies focused on the development of small molecule enzyme inhibitors of histone-modifying enzymes and HDAC inhibitor may reverse misregulated epigenetic states and has been seen as a promising therapy of cancer ${ }^{15}$.

Recently studies indicated that combined $\mathrm{PI} 3 \mathrm{~K} / \mathrm{mTOR}$ inhibitor with HDAC inhibitor may be more effective than a single drug intervention in various human tumor cells. Chen et al found co-treatment with BEZ235 and TSA enhanced autophagic cell death and blocked tumor growth without noticeable side effects in breast cancer 17 . Erlich et al. reported that the combination with the HDAC inhibitor Panobinostat and the PI3K/mTOR inhibitor BGT226 and BEZ235 could inhibit the viability of head and neck squamous carcinoma cells 
better than single drug ${ }^{18}$. Some previous studies had reported that HDAC inhibitor TSA could suppresses proliferation and promotes apoptosis of esophageal squamous cell lines ${ }^{29-31}$. But the effect of combination of HDAC inhibitors and PI3K/mTOR inhibitor on ESCCs is still unknown. Our recently study found co-treatment with BEZ235 and TSA could inhibit the growth and visibility in human esophageal cancer Eca-109 and TE-1 cells and enhanced autophagic cell death by up-regulating the expression of LC3-II and Beclin-1. Meanwhile, co-treatment with BEZ235 and TSA can increase the expression of cleaved caspase-3 and decrease the expression of caspase-3 and BCL-2. These results suggested that co-treatment with BEZ235 and TSA synergistically induced apoptosis and autophagy.

To our knowledge, this is the first study investigating the combination of mTOR and HDAC inhibitors in human esophageal squamous cell carcinoma cells. The previous studies revealed that HDAC inhibitors can potentially augment the anticancer activity of PI3K/mTOR pathway inhibition by further reducing AKT activation. Thus our finding suggested that compared with either drug alone, combining $\mathrm{PI} 3 \mathrm{~K} / \mathrm{mTOR}$ and HDAC-inhibitors can significantly inhibit the viability and induce the programmed cell death, such as autophagy and apoptosis in ESCC cells through the mechanistic convergence onto the $\mathrm{PI} 3 \mathrm{~K} / \mathrm{mTOR}$ pathway.

In summary, our study showed the Beclin-1 and LC3 were lower in ESCC tissues than adjacent normal tissues, and the high expression of Beclin-1 and LC3 were favorable prognostic indicators of overall survival. The activation of the PI3K/mTOR pathway is observed in ESCCs compared with normal esophageal mucosa, which can block autophagy and induce the decreasing expressions of autophagyrelated proteins such as Beclin-1, LC3-II and the ratio of LC3-II/LC3-I. Co-treatment with BEZ235 and TSA is more effective than single drug in inhibiting the viability of ESCC cells in Vitro through inducing apoptosis and autophagy, which can be a new effective therapeutic target in ESCCs.

\section{Abbreviations}

AJCC: American Joint Committee on Cancer; CI: confidence interval; HR: hazard ratio; OS: overall survival; DFS: disease-free survival; ESCC: esophageal squamous cell carcinoma; EAC: esophageal adenocarcinoma; HDAC: histone deacetylase; TSA: Trichostatin A; PCD: Programmed cell death; PI3K/mTOR: phosphoinositide 3-kinase $(\mathrm{PI} 3 \mathrm{~K}) /$ mammalian target of rapamycin.

\section{Acknowledgements}

This work was supported by grant from the Shanghai Municipal Health \& Family Planning Commission (No. 20174Y0238).

\section{Competing Interests}

The authors have declared that no competing interest exists.

\section{References}

1. Torre LA, Bray F, Siegel RL, et al. Global cancer statistics. CA Cancer J Clin. 2015; 65:87-108.

2. Chen $W$, Zheng R, Zhang S, et al. Esophageal cancer incidence and mortality in China, 2010. Thorac Cancer. 2011; 5: 343-8.

3. $\mathrm{Wu} \mathrm{N}, \mathrm{Chen} \mathrm{G}, \mathrm{Hu} \mathrm{H}$, et al. Low pretherapeutic serum albumin as a risk factor for poor outcome in esophageal squamous cell carcinomas. Nutr Cancer. 2015; 67: 481-485.

4. Wu N, Chen Z, Pang L, et al. Prognostic significance of lymph node characteristics on survival in esophageal squamous cell carcinomas. Wien Klin Wochenschr. 2013; 125:26-33.

5. Wu N, Pang LW, Chen ZM, et al. Tumour length is an independent prognostic factor of esophageal squamous cell carcinomas. Chinese Medical Journal, 2012; 125: 4445-4448.

6. Ouyang L, Shi Z, Zhao S, et al. Programmed cell death pathways in cancer: a review of apoptosis, autophagy and programmed necrosis. Cell Prolif. 2012; 45: 487-98.

7. Yu S, Li G, Wang Z, et al. Low expression of MAP1LC3B, associated with low Beclin-1, predicts lymph node metastasis and poor prognosis of gastric cancer. Tumour Biol. 2016; 37:15007-15017.

8. Jiang ZF, Shao LJ, Wang WM, et al. Decreased expression of Beclin-1 and LC3 in human lung cancer. Mol Biol Rep. 2012; 39:259-67.

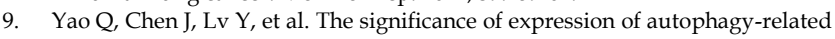
gene Beclin, Bcl-2, and Bax in breast cancer tissues. Tumour Biol. 2011; 32: $1163-71$

10. YS Chen, Y Lu, CL Lu, et al. Beclin-1 Expression is a Predictor of Clinical Outcome in Patients with Esophageal Squamous Cell Carcinoma and Correlated to Hypoxia-Inducible Factor (HIF)-1a Expression. Pathol Oncol Res. 2009; 15: 487-493.

11. Chen Y, Li X, Wu X, et al. Autophagy-related proteins LC3 and Beclin-1 impact the efficacy of chemoradiation on esophageal squamous cell carcinoma. Pathol Res Pract. 2013; 209:562-7.

12. Li B, Xu WW, Lam AKY, et al. Significance of PI3K/AKT signaling pathway in metastasis of esophageal squamous cell carcinoma and its potential as a target for anti-metastasis therapy. Oncotarget. 2017; 8:38755-38766.

13. Lin JW, Li X, Qiu ML, et al. PI3K Overexpression and PIK3CA Mutations Are Associated with Age, Tumor Staging, and Other Clinical Characteristics in Chinese Patients with Esophageal Squamous Cell Carcinoma. Genet Test Mol Biomarkers. 2017; 21: 236-241.

14. Xu DW, Zhang GQ, Wang ZW, et al. Autophagy in tumorigenesis and cancer treatment. Asian Pac J Cancer Prev. 2015; 16: 2167-75.

15. Mottamal M, Zheng S, Huang TL, et al. Histone deacetylase inhibitors in clinical studies as templates for new anticancer agents. Molecules. 2015; 20: 3898-3941.

16. Lakshmaiah KC, Jacob LA, Aparna S, et al. Epigenetic therapy of cancer with histone deacetylase inhibitors. J Cancer Res Ther. 2014; 10: 469-78.

17. Chen L, Jin T, Zhu K, et al. PI3K/mTOR dual inhibitor BEZ235 and histone deacetylase inhibitor Trichostatin A synergistically exert anti-tumor activity in breast cancer. Oncotarget. 2017; 8:11937-11949.

18. Erlich RB, Kherrouche Z, Rickwood D, et al. Saunders NA. Preclinical evaluation of dual PI3K-mTOR inhibitors and histone deacetylase inhibitors in head and neck squamous cell carcinoma. Br J Cancer. 2012; 106: 107-15.

19. Piao J, Chen L, Quan T, et al. Superior efficacy of co-treatment with the dual $\mathrm{PI} 3 \mathrm{~K} / \mathrm{mTOR}$ inhibitor BEZ235 and histone deacetylase inhibitor Trichostatin A against NSCLC. Oncotarget. 2016; 7: 60169-60180.

20. Rice TW, Patil DT, Blackstone EH. 8th edition AJCC/UICC staging of cancers of the esophagus and esophagogastric junction: application to clinical practice. Ann Cardiothorac Surg. 2017; 6:119-130.

21. Macintosh RL, Ryan KM. Autophagy in tumour cell death. Semin Cancer Biol. 2013; 23:344-351.

22. Fu LL, Cheng B, Liu B. Beclin-1: Autophagic regulator and therapeutic target in cancer. Int J Biochem Cell Biol. 2013; 45: 921-924.

23. Tanida I, Ueno T, Kominami E. LC3 conjugation system in mammalian autophagy. Int J Biochem Cell Biol. 2004; 36:2503-18.

24. Klionsky DJ, Abdelmohsen K, Abe A, et al. Guidelines for the use and interpretation of assays for monitoring autophagy (3rd edition). Autophagy. 2016; 12: 1-222.

25. Yang Z, Ghoorun RA, Fan X, et al. High expression of Beclin-1 predicts favorable prognosis for patients with colorectal cancer. Clin Res Hepatol Gastroenterol. 2015; 39:98-106. 
26. $\mathrm{SH} \mathrm{Wu}, \mathrm{CB}$ Sun, D Tian, et al. Expression and clinical significances of Beclin1, LC3 and mTOR in colorectal cancer. Int J Clin Exp Pathol. 2015; 8: 3882-3891.

27. Weh KM, Howell AB, Kresty LA. Expression, modulation, and clinical correlates of the autophagy protein Beclin-1 in esophageal adenocarcinoma. Mol Carcinog. 2016; 55:1876-1885.

28. Roesly HB, Khan MR, Chen HD, et al. The decreased expression of Beclin-1 correlates with progression to esophageal adenocarcinoma: the role of deoxycholic acid. Am J Physiol Gastrointest Liver Physiol. 2012; 302: 864-72.

29. Ma J, Guo X, Zhang S, et al. Trichostatin A, a histone deacetylase inhibitor, suppresses proliferation and promotes apoptosis of esophageal squamous cell lines. Mol Med Rep. 2015; 11:4525-31.

30. Ahrens TD, Timme S, Hoeppner J, et al. Selective inhibition of esophageal cancer cells by combination of HDAC inhibitors and Azacytidine. Epigenetics. 2015; 10: 431-445.

31. Zhou F, Li YH, Wang JJ, et al. Endoplasmic reticulum stress could induce autophagy and apoptosis and enhance chemotherapy sensitivity in human esophageal cancer EC9706 cells by mediating PI3K/Akt/mTOR signaling pathway. Tumour Biol. 2017; 39: 1010428317705748. 